\title{
DESMOCERÁTIDOS (AMMONOIDEA) DEL APTIENSE INFERIOR (CRETÁCICO INFERIOR) DE LA SUBCUENCA DE OLIETE, CORDILLERA IBÉRICA ORIENTAL (TERUEL, ESPAÑA)
}

\author{
Antoni GRAUGES ${ }^{1}$, Josep Anton MORENO- \\ BEDMAR ${ }^{2}$ y Ricardo MARTÍNEZ ${ }^{1}$
}

${ }^{1}$ Departament de Geologia (Paleontologia). Universitat Autònoma de Barcelona.
Edifici C. 08193-Bellaterra, (Barcelona) España. antonigrauges@hotmail.com,
Ricard.Martinez@uab.cat
${ }^{2}$ Departament de Geoquímica, Petrologia i Prospecció Geològica, Universitat de
Barcelona. Martí i Franquès s/n, 08028 Barcelona, España. j.a.moreno@ub.edu

Grauges, A., Moreno-Bedmar, J. A. \& Martínez, R. 2010. Desmocerátidos (Ammonoidea) del Aptiense Inferior (Cretácico Inferior) de la subcuenca de Oliete, Cordillera Ibérica Oriental (Teruel, España). [Lower Aptian (Lower Cretaceous) desmoceratids (Ammonoidea) of the Oliete sub-basin, Iberian Range (Teruel, Spain).] Revista Española de Paleontología, 25 (1), 7-18. ISSN 0213-6937.

\begin{abstract}
This study focuses on the lower Aptian desmoceratid fauna of Oliete sub-basin (Teruel province). The systematic revision of these biostratigraphy well constrained ammonites, allowed us to work on two issues.

On the fist place, we improved the systematic and taxonomic knowledge about Pseudosaynella raresulcata (d'Orbigny, 1841), Pseudosaynella bicurvata (Michelin, 1838) and Pseudohaploceras liptoviensis (Zeuschner, 1856) whose definitions, previous to this study, were based on scarce material and few studies on their ontogenetic variation. On a second place, we constrained their stratigraphical ranges, especially for the Pseudosaynella species, and their correlation with the lower Aptian standard ammonite Mediterranean zonation.
\end{abstract}

Keywords: lower Aptian, ammonoids, systematics, biostratigraphy, Oliete sub-basin, Iberian Chain, Teruel, Spain.

\section{RESUMEN}

Este estudio trata de la fauna de desmocerátidos del Aptiense inferior de la subcuenca de Oliete (provincia de Teruel). La revisión taxonómica de estos ammonites, bien situados bioestratigráficamente, permite dos precisiones.

La definición de Pseudosaynella raresulcata (d'Orbigny, 1841), Pseudosaynella bicurvata (Michelin, 1838) y de Pseudohaploceras liptoviensis (Zeuschner, 1856) se realizó a partir de pocos ejemplares, sin tener en cuenta sus variaciones ontogenéticas. En este trabajo se amplía el conocimiento de estas especies desde el punto de vista sistemático y taxonómico.

En segundo lugar se precisa la posición bioestratigráfica, en especial de las especies del género Pseudosaynella, correlacionando su distribución estratigráfica con las biozonas de ammonites estándar de la provincia faunística Mediterránea para el Aptiense inferior.

Palabras clave: Aptiense inferior, ammonites, sistemática, bioestratigrafía, subcuenca de Oliete, Cordillera Ibérica, Teruel, España.

\section{INTRODUCCIÓN}

Las especies de desmocerátidos del Aptiense inferior pertenecientes a los géneros Pseudosaynella Spath, 1923 y Pseudohaploceras Hyatt, 1900 fueron definidas sin tener en cuenta las variaciones ontogenéticas. Las descripciones de las especies europeas se han basado en unos pocos ejemplares, generalmente formas de pequeño tamaño, como ya apuntó en su día Imlay (1945: 280) para las Pseudosaynella, y por tanto su ontogenia y su variabilidad son poco conocidas. 
La subcuenca de Oliete ofrece abundancia de ejemplares de Pseudosaynella raresulcata (d'Orbigny, 1841) y P. bicurvata (Michelin, 1858), permitiéndonos apreciar la variación morfológica de cada una de estas especies en sus distintos estadios ontogenéticos, en especial la forma de la sección y su ornamentación.

Por otra parte, en la subcuenca de Oliete y áreas próximas, el registro bioestratigráfico de los ammonites estudiado por Murat (1983), Martínez et al. (1994), Moreno et al. (2007), Moreno-Bedmar et al. (2009, en prensa), está basado en los deshayesítidos que son clásicamente los ammonites empleados para las biozonas del Aptiense inferior. En este trabajo se correlaciona la distribución bioestratigráfica de las especies estudiadas con las biozonas ya establecidas en la provincia faunística Mediterránea por Reboulet et al. (2009).

\section{ANTECEDENTES}

El registro de cefalópodos aptienses de la zona es conocido desde antiguo (Vilanova, 1870); sin embargo los desmocerátidos no fueron citados hasta el trabajo de Sornay \& Marin (1972) donde describen la nueva especie Pseudosaynella josaensis. Posteriormente Murat (1983) cita y figura Pseudosaynella josaensis y Pseudohaploceras cf. liptoviense (Zeuschner, 1856).

Martínez et al. (1994), Moreno et al. (2007) y Moreno-Bedmar et al. (en prensa), citan y figuran también algunas especies de Pseudosaynella como P. undulata (Sarasin, 1893), $P$. raresulcata (d'Orbigny, 1841) y $P$. bicurvata (Michelin, 1838), pero sin descripción morfológica.

\section{ENCLAVE GEOGRÁFICO Y GEOLÓGICO}

La subcuenca de Oliete es una de las siete subcuencas que integran la Cuenca del Maestrazgo (Salas \& Guimerà, 1996) que se desarrolló durante la segunda etapa de rift del Jurásico superior-Cretácico inferior relacionada con la apertura del Atlántico central y el Atlántico norte (Salas et al., 2001). La subcuenca de Oliete está situada en la parte noroccidental de la cuenca del Maestrazgo en la Rama Aragonesa de la Cordillera Ibérica (Fig. 1). Limita al norte y noreste con la Cuenca Terciaria del Ebro, al este con la Depresión Terciaria de Calanda, al oeste con el anticlinal de Montalbán, al sur con la subcuenca de Aliaga y al Sudeste con la subcuenca de Morella. La subcuenca de Oliete se formó en el bloque superior hundido de una falla lístrica que fue especialmente activa durante el Aptiense inferior.

Los ammonoideos estudiados proceden de la Formación Margas del Forcall (Canérot et al., 1982: 286-288), que está constituida por margas con intercalaciones calizas que se hacen más abundantes hacia el techo. La edad

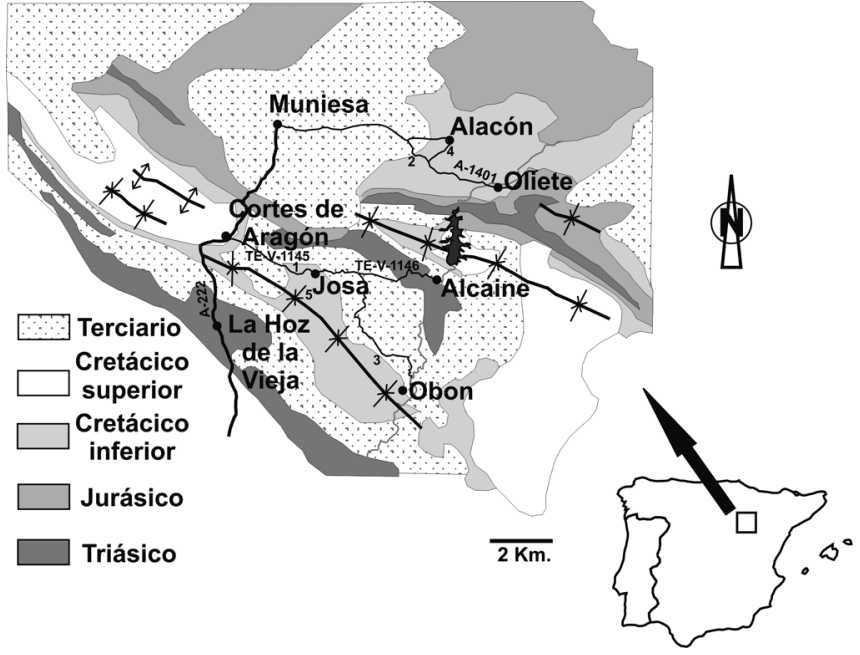

Figura 1. Mapa geológico simplificado con la situación de los yacimientos citados: 1, La Tejería de Josa; 2, Km. 8,6 de la carretera de Oliete-Muniesa; 3, Ermita de San Miguel; 4, Solana Emilia; 5, La Plana.

Geologic simplified map showing the situation of the localities: 1, La Tejería de Josa; 2, Km. 8.6 de la carretera de Oliete-Muniesa; 3, Ermita de San Miguel; 4, Solana Emilia; 5, La Plana.

de esta formación en la subcuenca de Oliete ha sido recientemente precisada por Moreno et al. (2007) y Moreno-Bedmar et al. (en prensa) que las atribuyen a la parte media-alta de la biozona Deshayesites forbesi, horizonte de Roloboceras hambrovi. La mayoría de los ammonites estudiados proceden de esta formación, de la localidad clásica de Josa (La Tejería) y de los alrededores de Josa: Obón, Alcaine y Alacón.

\section{ABREVIATURAS Y CONVENCIONES}

\section{Colecciones:}

A: Colección Albert Casasús. Barcelona.

AG: Colección Antoni Grauges. Caldes de Montbui (Barcelona).

JM: Colección José María Quer. Barcelona.

MSB: Museo de Geología del Seminario Conciliar de Barcelona.

PUAB: Colecciones de Paleontología de la Universitat Autònoma de Barcelona.

Medidas:

D: Diámetro de la concha.

H: Altura de la vuelta.

E: Anchura de la vuelta.

O: Diámetro del ombligo.

H/D: Grado de enrollamiento

E/D: Grado de aplanamiento

O/D: Amplitud ombilical

E/H: aplanamiento total 


\section{SISTEMÁTICA}

Clase CEPHALOPODA Cuvier, 1795

Orden AMMONITIDA Zittel, 1884

Superfamilia Desmoceratoidea Zittel, 1895

Familia Desmoceratidae Zittel, 1895

Subfamilia Pseudosaynellinae Casey, 1961

Género Pseudosaynella Spath, 1923

\section{Pseudosaynella raresulcata (d'Orbigny, 1841)} Fig. 2 g-n

1841 Ammonites raresulcatus Leymerie; d'Orbigny, 288, lám. 85, figs. 5-7.

1841 Ammonites raresulcatus; Leymerie, 15, lám. 17, fig. 20a-b.
1893 Sonneratia undulata; Sarasin, 162, fig. 11; lám. 4, fig. 8a; lám. 5, fig. 8b.

1893 Sonneratia raresulcata (Leymerie); Sarasin, 161, fig. 7; lám. 4, figs. 4a, 5a, 6a; lám. 5, figs. 4b, 5b, 6b; lám. 6, fig. c.

? 1893 Sonneratia heimi; Sarasin, 162, fig. 10; lám. 4, fig. $7 \mathrm{a}$; lám. 5, fig. $7 \mathrm{~b}$.

? 1906 Sonneratia cf. raresulcata d'Orbigny; Douvillé, láms. 13, fig. 2, 2a.

1960 Pseudosaynella undulata (Sarasin); Waitzman, 53, lám. 2, fig. 11a-b; lám. 13, figs. 8-11.

1960 Pseudosaynella raresulcata (Leymerie); Waitzman, 50, lám. 2, fig. 10a-b.; lám. 13, figs. 1-7.

1960 Pseudosaynella raresulcata (Leymerie); Drushchits, 303, lám. 45, fig. 3a-b.

1961 Pseudosaynella raresulcata (Leymerie); Casey, 171, fig. 53d-e.

Tabla 1. Medidas de Pseudosaynella raresulcata (d'Orbigny, 1841). En la parte superior de la tabla las tomadas de la bibliografía y en la parte inferior los ejemplares de este trabajo.

Measurements of Pseudosaynella raresulcata (d'Orbigny, 1841), in the upper half of the table those taken from the literature and in the lower half the specimens of this paper.

\begin{tabular}{|c|c|c|c|c|c|}
\cline { 2 - 6 } \multicolumn{1}{c|}{} & $\mathrm{D}$ & $\mathrm{H}(\mathrm{H} / \mathrm{D})$ & $\mathrm{E}(\mathrm{E} / \mathrm{D})$ & $\mathrm{O}(\mathrm{O} / \mathrm{D})$ & $\mathrm{E} / \mathrm{H}$ \\
\hline Sarasin (1893) fig. 4 a-b & 34.5 & $17.5(0.51)$ & $10.5(0.30)$ & $5.5(0.16)$ & 0.60 \\
\hline Sarasin (1893) fig. 8 a-b & 26.5 & $14.0(0.53)$ & $8.5(0.32)$ & $5.5(0.21)$ & 0.57 \\
\hline Waitzman (1960) jac. 4 & 29.9 & $14.6(0.49)$ & $8.0(0.27)$ & $4.2(0.14)$ & 0.55 \\
\hline $\begin{array}{c}\text { Martínez (1982) } \\
\text { PUAB 5276b }\end{array}$ & 22.2 & $11.7(0.53)$ & $6.5(0.30)$ & $3.5(0.16)$ & 0.55 \\
\hline $\begin{array}{c}\text { Martínez (1982) } \\
\text { PUAB 5284a }\end{array}$ & 21.2 & $11.5(0.55)$ & $6.8(0.33)$ & $3.8(0.18)$ & 0.59 \\
\hline $\begin{array}{c}\text { Martínez (1982) } \\
\text { PUAB 5284b }\end{array}$ & 16.0 & $7.5(0.47)$ & $5.5(0.34)$ & $2.8(0.17)$ & 0.73 \\
\hline \hline $\begin{array}{c}\text { Fischer \& Gauthier (2006) } \\
\text { neotipo LPMP-R4314 }\end{array}$ & 15.0 & $8.0(0.53)$ & $5.5(0.37)$ & $3.0(0.20)$ & 0.61 \\
\hline A-90 & - & $23.4(-)$ & $13.6(-)$ & $-(-)$ & 0.58 \\
\hline PUAB 5910 & 26.4 & $13.2(0.50)$ & $7.1(0.27)$ & $-(-)$ & 0.53 \\
\hline A-100 & 28.0 & $14.0(0.50)$ & $8.0(0.28)$ & $6.0(0.21)$ & 0.57 \\
\hline AG-20 & 41.2 & $22.4(0.54)$ & $10.4(0.25)$ & $6.7(0.16)$ & 0.46 \\
\hline A-105 & 51.0 & $22.5(0.44)$ & $-(-)$ & $16.6(0.20)$ & - \\
\hline A-67 & 61.0 & $32.0(0.52)$ & $14.0(0.23)$ & $9.3(0.15)$ & 0.43 \\
\hline PUAB 48412 & 62.2 & $35.0(0.56)$ & $16.0(0.25)$ & $8.5(0.13)$ & 0.45 \\
\hline JM-02 & 87.5 & $46.0(0.52)$ & $23.5(0.26)$ & $12.6(0.14)$ & 0.51 \\
\hline JM-23 & 92.2 & $49.2(0.53)$ & $22.5(0.24)$ & $12.0(0.14)$ & 0.45 \\
\hline A-07 & $50.5(0.53)$ & $23.8(0.25)$ & $11.3(0.12)$ & 0.47 \\
\hline A-36 & $59.0(0.53)$ & $25.8(0.23)$ & $12.9(0.11)$ & 0.43 \\
\hline
\end{tabular}



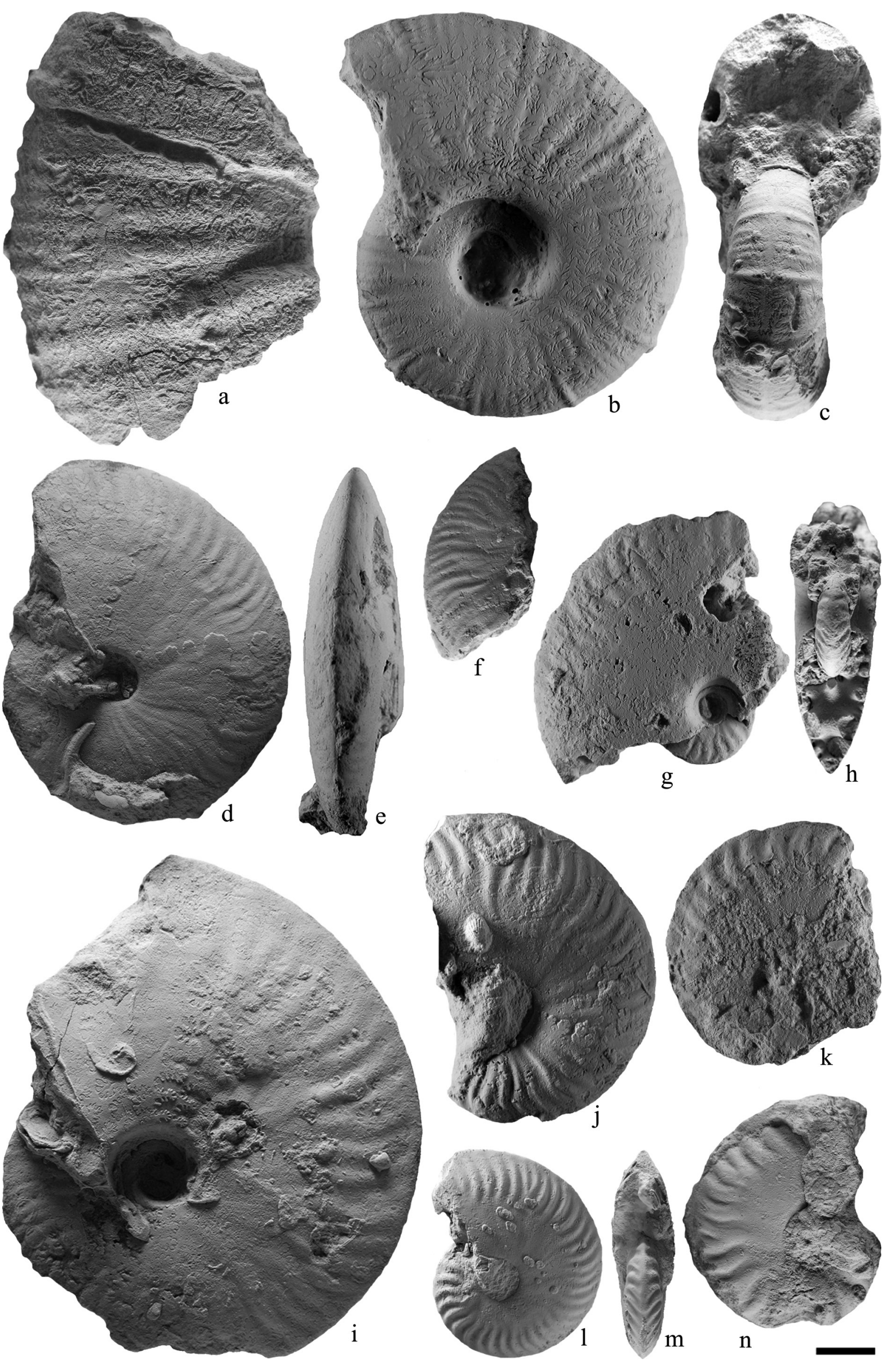
1961 Pseudosaynella undulata (Sarasin); Casey, 171, fig. 53f.

1961 Pseudosaynella aff. undulata (Sarasin); Casey, 173, lám. 29, fig. 4a-c.

1961 Pseudosaynella cf. fimbrita (Imlay); Casey, 172, lám. 29, fig. 3a-d, fig. 53j.

1972 Pseudosaynella josaensis Sornay \& Marin, 111, textfigs. 10-11; lám. D, figs. 2-3.

v 1982 Pseudosaynella undulata (Sarasin); Martínez, 61, lám. 3, figs. 7a, 8a, 9a-c.

v 1982 Pseudosaynella raresulcata (Leymerie); Martínez, 59, lám. 3, figs. 4a, 5a-b, 6a-c.

1983 Pseudosaynellajosaensis Sornay; Murat, lám. 7, figs. 2-3.

? 2004 Pseudosaynella ex gr. undulata (Sarasin); Bogdanova \& Hoedemaeker, 218; lám. 28, fig. 3a-c.

2004 Pseudosaynella raresulcata (d'Orbigny); Amédro \& Matrion, 79, lám. 1, figs. 4a-b, 5a-b.

2006 Pseudosaynella raresulcata (d’Orbigny); Guérin-Franiatte (in Fischer \& Gauthier), 106, lám. 30, fig. 6a-c.

pars 2006 Pseudosaynella bicurvata (d'Orbigny); Busnardo \& Gauthier (in Fischer \& Gauthier), 104, lám. 30, fig. 2a-c. Non fig. 3 (=P. bicurvata).

v 2007 Pseudosaynella undulata (Sarasin); Moreno et al., 77, lám. 1, fig. 10.

v 2007 Pseudosaynella bicurvata (Sarasin); Moreno et al., 77, lám. 1, fig. 3 .

2008 Pseudosaynella raresulcata (Leymerie); Abu-Zied, 606, figs. 2O, 6F-H.

Neotipo: Por designación de Guérin-Franiatte (in Fischer \& Gauthier, 2006), el ejemplar número LPMP-R4314 (5593 A-1 de la colección d'Orbigny), figurado en la lám. 30, fig. 6a-c; procedente del Aptiense inferior de Gurgy, Yonne (Francia), depositado en el Muséum National d'Histoire Naturelle de Paris.

Material: PUAB: 5907-5910, 5912-5914, 48001, 48014, 48015, 48021, 48058, 48060, 48097, 48118, 48120, 48132, 48137, 48142, 48149, 48155, 48159, 48196, 48200, 48208, 48210, 48230, 48247, 48284, 48319, 48385, 48412-48414. AG: 15, 20 , 107. JM: $2,17,20,23$. A: 7, 15, 24, 25, 31, 32, 36, 37, 40-43,
45-49, 52-60, 62-64, 67, 68, 70, 72, 74-76, 78, 80-83, 85-88, 90-93, 95, 96, 98, 99-101, 103, 104-110. MGSB: 27536, 46659.

La mayoría de ellos se encuentran fragmentados y son moldes internos calcáreos. Los ejemplares PUAB-48208, 48247 están limonitizados. Algunos presentan epifauna de ostréidos y serpúlidos; también los hay que presentan deformaciones o están parcialmente aplastados.

Dimensiones: Tabla 1.

Descripción: Concha discoidal muy involuta y de crecimiento rápido. La sección de la vuelta es ojival. El ombligo es estrecho, profundo y de paredes verticales. Los flancos son convexos, formando un vientre agudo.

Se distinguen tres estadios de crecimiento en los cuales cambia su morfología.

- Hasta un diámetro variable que oscila aproximadamente entre los 25 y $35 \mathrm{~mm}$, la ornamentación está constituida por costillas anchas y gruesas de tipo falcoide que ocupan casi todo el flanco sin atravesar el vientre, algunas de estas costillas se

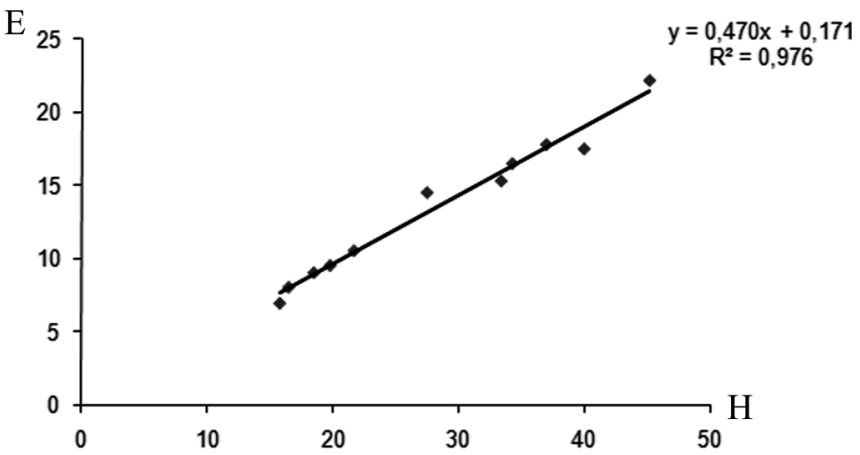

Figura 3. Relación E/H de Pseudosaynella raresulcata (d'Orbigny, 1841), según datos de la tabla 1. E/H relation of Pseudosaynella raresulcata (d'Orbigny, 1841), after table 1 data.

Figura 2. a: Pseudohaploceras liptoviensis (Zeuschner, 1856) vista lateral del ejemplar PUAB 48190. b-c: Pseudohaploceras liptoviensis (Zeuschner, 1856) vista lateral y ventral del ejemplar PUAB 48411. d-e: Pseudosaynella bicurvata (Michelin, 1838) vista lateral y ventral del ejemplar PUAB 48412. f: Pseudosaynella bicurvata (Michelin, 1838) vista lateral del ejemplar PUAB 5911. g-h: Pseudosaynella raresulcata (d'Orbigny, 1841) vista lateral y de la sección de la vuelta del ejemplar PUAB 48247. i: Pseudosaynella raresulcata (d'Orbigny, 1841) vista lateral del ejemplar PUAB 48137. j: Pseudosaynella raresulcata (d'Orbigny, 1841) vista lateral del ejemplar PUAB 48413. k: Pseudosaynella raresulcata (d'Orbigny, 1841) vista lateral del ejemplar PUAB 48414. l-m: Pseudosaynella raresulcata (d'Orbigny, 1841) vista lateral y en sección del ejemplar PUAB 48385. n: Pseudosaynella raresulcata (d'Orbigny, 1841) vista lateral del ejemplar PUAB 48319. Escala gráfica $1 \mathrm{~cm}$.

$\boldsymbol{a}$ : Pseudohaploceras liptoviensis (Zeuschner, 1856) lateral view of the specimen PUAB 48190. b-c: Pseudohaploceras liptoviensis (Zeuschner, 1856) lateral and ventral view of the specimen PUAB 48411. d-e: Pseudosaynella bicurvata (Michelin, 1838) lateral and ventral view of the specimen PUAB 48412. $\mathrm{f}$ : Pseudosaynella bicurvata (Michelin, 1838) lateral view of the specimen PUAB 5911. $\boldsymbol{g}$ - $\boldsymbol{h}$ : Pseudosaynella raresulcata (d'Orbigny, 1841) lateral and section of the whorl view of the specimen PUAB 48247. $i$ : Pseudosaynella raresulcata (d'Orbigny, 1841) lateral view of the specimen PUAB 48137. j: Pseudosaynella raresulcata (d'Orbigny, 1841) lateral view of the specimen PUAB 48413. $\boldsymbol{k}$ : Pseudosaynella raresulcata (d'Orbigny, 1841) lateral view of the specimen PUAB 48414. l-m: Pseudosaynella raresulcata (d'Orbigny, 1841) lateral and section of the whorl view of the specimen PUAB 48385. $\boldsymbol{n}$ : Pseudosaynella raresulcata (d'Orbigny, 1841) lateral view of the specimen PUAB 48319. Scale bar $1 \mathrm{~cm}$. 
bifurcan a mitad del flanco. Se aprecian entre 8 y 9 costillas por cada cuarto de vuelta.

- Entre los $35 \mathrm{~mm}$ y $75 \mathrm{~mm}$ de altura de vuelta (H), la sección de vuelta se estiliza, disminuyendo ligeramente la relación E/D. Las costillas son menos prominentes, al principio van desde el borde del ombligo hasta el vientre y posteriormente ocupan sólo los dos tercios superiores del flanco, aumentando su densidad a 14 costillas por $1 / 4$ de vuelta entre $\mathrm{H}=35$ y $\mathrm{H}=43$. $\mathrm{Al}$ final de este estadio las costillas son cada vez menos visibles y ocupan solamente el tercio superior del flanco.

- Alrededor de $\mathrm{H}=75$ desaparece la ornamentación, dificultando la comparación de ésta con otras especies de este género.

Discusión: En los trabajos de Waitzman (1960) y Martínez (1982) se observa que los ejemplares allí descritos de $P$. undulada (Sarasin, 1893) son de pequeño tamaño y los de $P$. raresulcata (d'Orbigny, 1841) son un poco mayores. En nuestro trabajo observamos que en ejemplares fragmentados como PUAB-48247 (Fig. 2 g-h), en sus estadios iniciales presentan características propias de $P$. undulata (formas con sección ancha y costillas muy robustas que ocupan todo el flanco) mientras que en estadios posteriores son claramente las de $P$. raresulcata (sección más estilizada, costillas menos prominentes y que no ocupan toda la altura del flanco), todo ello en el mismo ejemplar; tenemos pues que las distintas morfologías atribuidas a raresulcata y undulata son en realidad variaciones ontogenéticas cuando comparamos series de individuos bien desarrollados.

Dentro de la variedad intraespecífica se observan ejemplares con la ornamentación más marcada y con el vientre más agudo, parecido a una quilla incipiente y otros con la sección de la vuelta ligeramente más estrecha y con la costulación más suave. Esto se observa tanto en los ejemplares estudiados en este trabajo (PUAB-48210, 48385, Fig. 2 l-m) como en los figurados por Sarasin (1893: láms. 4, fig. 4a; lám. 5, fig. 4b), Casey (1961: lám. 29, fig. 3a-d), Martínez (1982: lám. 3, fig. 6b-c), Guérin-Franiatte (in Fischer \& Gauthier, 2006: lám. 30, fig. 2a-c).

La especie descrita por Sornay \& Marin (1972) en La Tejería de Josa, $P$. josaensis no presenta variaciones con respecto a $P$. raresulcata. Sornay \& Marin (1972) compararon sus ejemplares con las especies norteamericanas de Arkansas $P$. fimbriata Imlay, 1945 y P. walcotti (Hill, 1888), pero no con la especie que nos ocupa.

Para la descripción de $P$. fimbriata, Imlay se basa unicamente en tres moldes internos calcáreos con apenas ornamentación visible excepto uno de ellos (Imlay, 1945: lám. 42, fig. 2). Este ejemplar de $78 \mathrm{~mm}$ de diámetro muestra una gran afinidad con ejemplares de un tamaño similar atribuibles a Pseudosaynella raresulcata. La especie $P$. walcotti, que presenta una sección algo más ancha que Pseudosaynella fimbriata y una densidad de costulación baja con unas costillas robustas, también muestra afinidad con $P$. raresulcata. Aun así no se incluyen en sinonimia ya que estas especies están descritas a partir de pocos ejemplares, sin conocer su ontogenia y variabilidad y pertenecen además en una provincia faunística diferente. Se desconoce también su posición respecto a las biozonas estándar de ammonites.

Distribución: Francia: "Argiles à Plicatules" de la Cuenca de París: Aptiense inferior, biozonas Deshayesites forbesi y Deshayesites deshayesi (Casey, 1961; Amédro \& Matrion, 2004; Guérin-Franiatte y Busnardo \& Gauthier in Fischer \& Gauthier, 2006).

España: Cuenca del Maestrazgo: Aptiense (Waitzman, 1960). Parte alta del Aptiense inferior (Marin \& Sornay, 1971; Sornay \& Marin, 1972). Bedouliense inferior, biozona Deshayesites forbesi (ver Murat, 1983). Aptiense inferior, biozona Deshayesites weissi (=D. forbesi) (ver Moreno et al., 2007). Cuenca d'Organyà: Aptiense inferior, biozona Deshayesites deshayesi (ver Martínez, 1982).

Colombia: Aptiense inferior (Bogdanova \& Hoedemaeker, 2004).

Egipto: Abu-Zied (2008) data el Aptiense medio por la presencia de Aconeceras nisus (biozona homónima). Creemos que en realidad se trata de un ejemplar de $P$. bicurva$t a$, forma característica del Aptiense inferior.

Cáucaso septentrional: Aptiense inferior del Cáucaso (Drushchits, 1960).

Yacimientos: La Tejería de Josa, Km. 8,6 de la carretera Oliete-Muniesa, Ermita de San Miguel, Solana Emilia, La Plana.

\section{Pseudosaynella bicurvata (Michelin, 1838)}

Fig. 2 d-f

1838 Ammonites bicurvatus Michelin, 101, lám. 12, figs.7, $7 \mathrm{a}$.

pars 1841 Ammonites bicurvatus Michelin; (d'Orbigny), 286, lám. 84, fig. 3. Non, lám. 84, figs. 1-2, 4 (=Cleoniceras cleon d'Orbigny).

? 1842 Ammonites bicurvatus Michelin; Chenu, lám. 12, fig. 1. non 1865 Ammonites bicurvatus Michelin; Trautschold, 22, lám. 3, fig. 17a-b, véase en Bogdanova \& Hoedemaeker (2004) (=Sinzovia trautscholdi Sinzow, holotipo).

non 1872 Ammonites bicurvatus Michelin; Tietze, 137, lám. 9, fig. 5a-b (=Barremites strettostoma Uhlig).

1893 Sonneratia bicurvata (Michelin); Sarasin, 161, textfigs. 8, 9, lám. 4, figs. 1a, 2a, 3a, lám. 5, figs. 1b, 2b, $3 \mathrm{~b}$.

1949 Saynella bicurvata (Michelin); Luppov et al., 244, text-fig. 74, lám. 74, fig. 1 a-c.

1958 Pseudosaynella bicurvata (Michelin); Orlov, lám. 51, fig. 2 a-b (=en traducción inglesa, Orlov, 1976)

1960 Pseudosaynella bicurvata (Michelin); Waitzman, 44, lám. 2, fig. 9a-b, láms. 6-12.

1960 Pseudosaynella bicurvata (Michelin); Drushchits, 302, text-fig. 88, lám. 45, fig. 2 a-b.

1961 Pseudosaynella bicurvata (Michelin); Casey, 171, fig. 53a-c. 
v 1975 Pseudosaynella aff. bicurvata (Michelin); Lillo, 686, lám. 4, figs. 7-8.

v 1982 Pseudosaynella bicurvata (Michelin); Martínez, 57, text-fig. 9; lám. 3, figs. 1a-b, 2a-b, 3a.

v 1994 Pseudosaynella bicurvata (Michelin); Martínez et al., lám. 1, fig. 1.

1999 Pseudosaynella bicurvata (Michelin); Bogdanova \& Prozorosvsky, lám. 7, figs. g, h.

2004 Pseudosaynella bicurvata (Michelin); Bogdanova \& Hoedemaeker, 217, lám. 28, figs. 1a-c, 2a-c.

2004 Pseudosaynella bicurvata (Michelin); Amédro \& Matrion, 79, lám. 1, fig. 6a-b.

pars 2006 Pseudosaynella bicurvata (d'Orbigny); Busnardo \& Gauthier (in Fischer \& Gauthier), 104, lám. 30, fig. 3. Non fig. 2 a-c (=P. raresulcata).

v 2007 Pseudosaynella raresulcata (Leymerie in d'Orbigny); Moreno et al., 77, lám. 1, fig. 12.

2008 Aconeceras nisus (d'Orbigny); Abu-Zied, 606, figs. 2I, $5 \mathrm{~K}-\mathrm{M}$.

Lectotipo: Por designación de Busnardo \& Gauthier (in Fischer \& Gauthier, 2006) el ejemplar número LPMP-B46132 (5597-
1 de la colección d'Orbigny) depositado en el Muséum National d'Histoire Naturelle de Paris, figurado en la lám. 30, fig. 3, procedente del Aptiense inferior de Villeneuve-Au-Chêne, Aube (Francia).

Material: PUAB: 5911, 48039, 48059, 48156, 48412; AG: 22, 25: JM: 12, 14, 18; A: 23, 33, 38, 44, 51, 61, 66, 69, 71, 73, 77, 79, 84, 89, 94, 102; MGSB: 24400. Muchos de ellos fragmentados. Están conservados en molde interno calcáreo.

Dimensiones: Tabla 2.

Descripción: Concha planiespiral muy involuta, sección de espira ojival, bastante comprimida. Los flancos son planos y se unen formando un vientre estrecho y agudo, sin quilla. El ombligo es estrecho y profundo, siendo su borde bastante anguloso. La ornamentación la forman costillas de tipo falcoide, muchas de las cuales son bifurcadas. Estas costillas son muy tenues, a veces casi inapreciables y ocupan solamente el tercio externo del flanco. Entre $\mathrm{H}=27 \mathrm{~mm}$ y $\mathrm{H}=33 \mathrm{~mm}$ se contabilizan unas 14 por cada cuarto de vuelta que no atraviesan el vientre.

Tabla 2. Medidas de Pseudosaynella bicurvata (Michelin, 1838). En la parte superior de la tabla las tomadas de la bibliografía y en la parte inferior los ejemplares de este trabajo.

Measurements of Pseudosaynella bicurvata (Michelin, 1838), in the upper half of the table those taken from the literature and in the lower half the specimens of this paper.

\begin{tabular}{|c|c|c|c|c|c|}
\hline & $\mathrm{D}$ & $\mathrm{H}(\mathrm{H} / \mathrm{D})$ & $E(E / D)$ & $\mathrm{O}(\mathrm{O} / \mathrm{D})$ & $\mathrm{E} / \mathrm{H}$ \\
\hline $\begin{array}{c}\text { Michelin (1838) lám. } 12 \\
\text { fig. } 7 \\
\end{array}$ & 30.0 & $16.5(0.55)$ & $8.0(0.27)$ & $4.0(0.14)$ & 0.48 \\
\hline Sarasin (1893) fig. $1 a-b$ & 51.0 & $27.5(0.54)$ & $14.5(0.28)$ & $5.5(0.11)$ & 0.52 \\
\hline Waitzman (1960) n 45 & 35.8 & $19.8(0.55)$ & $9.5(0.26)$ & $3.8(0.11)$ & 0.48 \\
\hline $\begin{array}{l}\text { Martínez (1982) } \\
\text { PUAB 5274a }\end{array}$ & 32.5 & $18.5(0.57)$ & $9.0(0.27)$ & $4.0(0.27)$ & 0.48 \\
\hline MGSB 24400 & 29.7 & $15.8(0.53)$ & $6.9(0.23)$ & $6.0(0.20)$ & 0.43 \\
\hline A-89 & 36.7 & $19.7(0.53)$ & $-(\longleftarrow)$ & $7.7(0.21)$ & - \\
\hline AG-25 & 42.8 & $25.5(0.59)$ & $-(\ldots)$ & $-(\ldots)$ & - \\
\hline A-38 & 50.5 & $27.2(0.53)$ & $-\left(\_\right)$ & $7.8(0.15)$ & - \\
\hline AG-31 & 57.5 & $33.4(0.58)$ & $15.3(0.27)$ & $8.9(0.15)$ & 0.46 \\
\hline PUAB 48039 & 60.0 & $32.4(0.55)$ & $-(\ldots)$ & $9.9(0.16)$ & - \\
\hline A-84 & 65.5 & $34.3(0.52)$ & $16.5(0.25)$ & $11.2(0.17)$ & 0.48 \\
\hline $\mathrm{JM}-12$ & - & $37.0\left(\_\right)$ & $17.8(\ldots)$ & $9.0\left(\_\right)$ & 0.48 \\
\hline JM-14 & - & $40.0(\ldots)$ & $17.5\left(\_\right)$ & $10.7\left(\_\right)$ & 0.43 \\
\hline A-33 & - & $45.2(\ldots)$ & $22.2\left(\_\right)$ & $-(\ldots)$ & 0.49 \\
\hline A-102 & - & $21.7\left(\_\right)$ & $10.8(\square)$ & $5.7(\ldots)$ & 0.49 \\
\hline
\end{tabular}




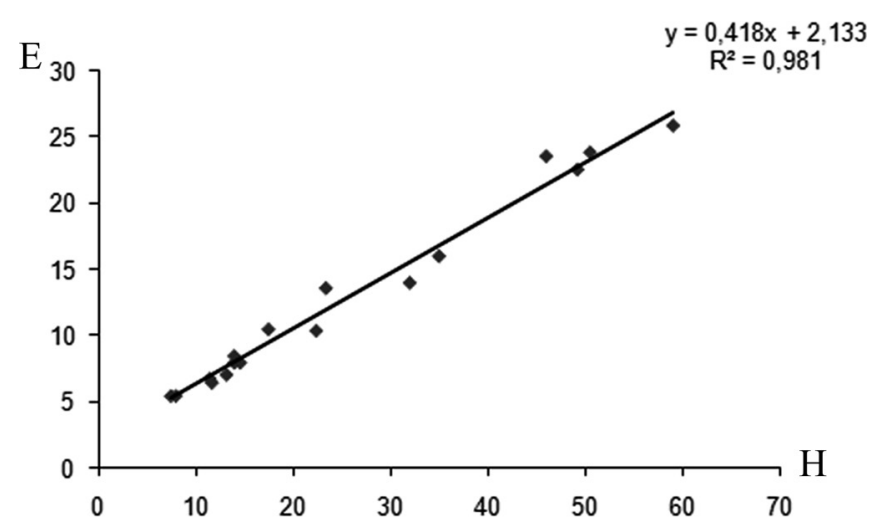

Figura 4. Relación E/H de Pseudosaynella bicurvata (Michelin, 1838), según datos de la tabla 2.

E/H relation of Pseudosaynella bicurvata (Michelin, 1838), after table 2 data.

Discusión: P. bicurvata (Michelin, 1838) tiene una densidad de costulación mayor que $P$. raresulcata (d'Orbigny, 1841) con costillas menos prominentes, en ocasiones apenas visibles y con una sección de la vuelta más estilizada. Durante su ontogenia la ornamentación varía poco y la costulación va atenuándose hasta desaparecer en torno a los $140 \mathrm{~mm}$ de diámetro.

Las formas con ornamentación menos visible se pueden confundir con especies del género Aconeceras Hyatt, 1903; diferenciándose estos últimos por tener una carena bien formada sobre un vientre más redondeado que en Pseudosaynella Spath, 1923. Estas mismas formas, sin apenas ornamentación, muestran también una gran similitud con algunas especies de Barremites Kilian, 1913. Dimitrova (1967) atribuye la especie B. strettostoma (Uhlig, 1883) al género Pseudosaynella. Los Barremites difieren esencialmente de las Pseudosaynella por presentar el vientre más redondeado.

Distribución: Francia: “Argiles à Plicatules" de la Cuenca de París: Aptiense (Amédro \& Matrion, 2004).

Cáucaso septentrional: Aptiense inferior del Cáucaso (Drushchits, 1960). Biozona Deshayesites deshayesi (Ap- tiense inferior) del Turkmenistan (Bogdanoba \& Prozorovsky, 1999).

Colombia: Aptiense inferior (Bogdanova \& Hoedemaeker, 2004).

España: Cuenca del Maestrazgo: Waitzman (1960) Aptiense. Biozona de Deshayesites weissi (=Deshayesites forbesi) (ver Moreno et al., 2007), Aptiense inferior. Cuenca d'Organyà: biozona Deshayesites deshayesi (ver Martínez, 1982), Aptiense inferior.

Egipto: Aptiense medio? de Egipto (Abu-Zied, 2008).

Yacimientos: La Tejería de Josa, Ermita de San Miguel.

\section{Pseudosaynella sp.}

Material: JM: 6; A: 28, 34, 35, 39, 50, 97. PUAB: 48002, 48006, 48007, 48030, 48032, 48148, 48153, 48161, 48230.

Dimensiones: Tabla 3.

Descripción: Se trata de ejemplares de gran talla en los que no se observa ningún tipo de ornamentación. La desaparición de la ornamentación, así como la similitud entre las mediciones de las distintas especies de Pseudosaynella de gran tamaño, no permiten atribuir estos ejemplares a ninguna especie en concreto.

Distribución: España: Cuenca del Maestrazgo, Biozona de Deshayesites weissi (=Deshayesites forbesi), Aptiense inferior.

Yacimientos: La Tejería de Josa, Ermita de San Miguel, La Plana.

Subfamilia Puzosiinae Spath, 1922

Género Pseudohaploceras Hyatt, 1900

\section{Pseudohaploceras liptoviense (Zeuschner, 1856)} Fig. 2 a-c

1856 Ammonites liptoviensis Zeuschner, 181, lám. 2, figs. 1a-b, 2a-b, 3a-b.

Tabla 3. Medidas de Pseudosaynella sp. Measurements of Pseudosaynella $s p$.

\begin{tabular}{|c|c|c|c|c|c|}
\cline { 2 - 6 } \multicolumn{1}{c|}{} & $\mathrm{D}$ & $\mathrm{H}(\mathrm{H} / \mathrm{D})$ & $\mathrm{E}(\mathrm{E} / \mathrm{D})$ & $\mathrm{O}(\mathrm{O} / \mathrm{D})$ & $\mathrm{E} / \mathrm{H}$ \\
\hline JM-02 & - & $46.0\left(\_\right)$ & $37.8\left(\_\right)$ & $25.2(0.14)$ & - \\
\hline A-28 & 215.0 & $115.0(0.53)$ & $34.0(0.15)$ & $24.0(0.11)$ & 0.29 \\
\hline A-39 & - & $101.5(\ldots)$ & $47.5\left(\_\right)$ & $\ldots(\ldots)$ & 0.46 \\
\hline A-50 & - & $121.7(\ldots)$ & $53.5(\ldots)$ & $\ldots(\ldots)$ & 0.44 \\
\hline
\end{tabular}


1868 Ammonites austeni Schloenbach, 465, lám. 11, fig. 3.

1883 Haploceras liptoviense (Zeuschner); Uhlig, 229, lám. 17, figs. 9, 16-18; lám. 18, figs. 1, 3, 5, 6.

1902 Desmoceras liptoviense (Zeuschner); Koenen, 62, lám. 43, fig. 1a-b.

1920 Puzosia liptoviensis (Zeuschner); Fallot, 259, text-fig. 21, lám. 1, figs. 5a-c, 6.

1933 Puzosia liptoviensis (Zeuschner); Rouchadzé, 182, lám. 2, fig. 3.

? 1938 Puzosia cf. liptoviensis (Zeuschner); Ksiąźkiewicz, 232, lám. 1, fig. 6 .

1972 Pseudohaploceras liptoviense (Zeuschner); Vašíček, 78, lám. 14, fig. 4.

V 1975 Pseudohaploceras liptoviensis (Zeuschner); Lillo, 684, lám. 2, figs. 8-9, lám. 3, figs. 1-2.

1982 Pseudohaploceras liptoviense (Zeuschner); Martínez, 67, lám. 4, figs. 6a, 7a, 8a-b, 9a-b.

1982 Pseudohaploceras liptovienses (Zeuschner); Braga et al., 695, lám. 1, fig. 2.

1983 Pseudohaploceras cf. liptoviense (Zeuschner); Murat, lám. 7, fig. 1.

1992 Pseudohaploceras liptoviense (Zeuschner); Delanoy, 31, lám. 4, figs. 5, 6a-b.

? 1996 Pseudohaploceras liptoviense (Zeuschner); GonzálezArreola et al., 38, lám. 4, figs. 1-9.

1997 Pseudohaploceras liptoviense (Zeuschner); Delanoy, lám. 2, fig. 6.

? 2005 Pseudohaploceras liptoviensis (Zeuschner); Dutour, 122, lám. 13, figs. 1a-c, 2a-d, 3a-c, 4a-d, 5a-f.

v 2007 Pseudohaploceras liptoviensis (Zeuschner); Moreno et al., lám. 1, fig. 11.

2009 Pseudohaploceras ex. gr. liptoviensis (Zeuschner); Lehmann et al., fig. $7 \mathrm{~L}$.

Lectotipo: según Vašíček (1972) el ejemplar figurado por Zeuschner (1856) depositado en el Museum für Geologische Bundesanstalt (Viena) procedente del Barremiense superior-Aptiense inferior de Lúčky, Mts. Chočské (Eslovaquia).
Material: PUAB: 48056, 48190, 48273, 48411. El ejemplar PUAB-48411 está bien conservado como molde interno calcáreo, muestra todas las líneas de sutura externas de la última vuelta y carece de cámara de habitación. Los demás ejemplares son fragmentos.

Dimensiones: Tabla 4.

Descripción: El ejemplar mejor conservado (PUAB-48411) muestra una concha globosa de sección bastante redondeada, medianamente evoluta, cada vuelta solapa la mitad de la altura de la anterior. Entre la cara del ombligo y el flanco forman un ángulo recto, de vértice redondeado.

La ornamentación consiste en costillas principales ligeramente flexuosas adoralmente, poco prominentes pero más marcadas y realzadas debido a la existencia de una ligera constricción a cada lado. Estas costillas van de borde umbilical a borde umbilical interrumpiéndose solamente sobre el sifúnculo. Hay 10 costillas principales en la última vuelta. Entre estas costillas principales hay otras, secundarias, entre 5 y 8 por cada par de principales, con tendencia a aumentar de número a medida que el tamaño crece y las principales se separan entre sí. Las costillas secundarias salen desde distintas alturas del flanco, algunas desde el borde del ombligo. La pared del ombligo es lisa.

Discusión: La costulación es tenue como la figurada por Fallot (1920) y por Lillo (1975), en cambio la figurada por Wright et al. (1996) es mucho más marcada.

El ejemplar PUAB-49190 tiene un tamaño enorme, en comparación con los ejemplares figurados hasta este momento.

P. matheroni, similar a esta especie, se distingue por tener la sección de vuelta más alta, midiéndose en el holotipo valores de $\mathrm{H} / \mathrm{D}=0,37$ y $\mathrm{E} / \mathrm{H}=0,28$. Recientemente Busnardo (in Fischer \& Gauthier, 2006) describe y figura el holotipo de $P$. matheroni (d'Orbigny, 1840) permitiendo diferenciar claramente esta especie de P. liptoviensis.

Tabla 4. Medidas de Pseudohaploceras liptoviensis (Zeuschner, 1856) extraídas de la bibliografía junto con las de los ejemplares estudiados en este trabajo.

Pseudohaploceras liptoviensis (Zeuschner, 1856) measurements taken from the literature, and the specimens of this work.

\begin{tabular}{|c|c|c|c|c|c|}
\cline { 2 - 6 } \multicolumn{1}{c|}{} & $\mathrm{D}$ & $\mathrm{H}(\mathrm{H} / \mathrm{D})$ & $\mathrm{E}(\mathrm{E} / \mathrm{D})$ & $\mathrm{O}(\mathrm{O} / \mathrm{D})$ & $\mathrm{E} / \mathrm{H}$ \\
\hline $\begin{array}{c}\text { Uhlig (1883) lám. 18 } \\
\text { fig. 1 }\end{array}$ & 75.0 & $33.0(0.44)$ & $-\left(\_\right)$ & $23.0(0.31)$ & - \\
\hline $\begin{array}{c}\text { Fallot (1920) lám. 1 } \\
\text { fig. 5 }\end{array}$ & 59.0 & $25.0(0.42)$ & $23.0(0.38)$ & $19.0(0.32)$ & 0.92 \\
\hline Vašíček (1972) KZ2/80 & 78.0 & $33.0(0.42)$ & $-\left(\_\right)$ & $21.0(0.27)$ & - \\
\hline Lillo (1975) 54A3b & 13.9 & $7.5(0.54)$ & $6.4(0.46)$ & $4.2(0.30)$ & 0.85 \\
\hline Lillo (1975) 54A3a & 19.4 & $8.0(0.41)$ & $7.7(0.39)$ & $4.5(0.23)$ & 0.96 \\
\hline \hline PUAB 48411 & 69.4 & $31.2(0.45)$ & $29.2(0.42)$ & $18.0(0.26)$ & 0.93 \\
\hline PUAB 48190 & - & $54.0\left(\_\right)$ & $-\left(\_\right)$ & $-(\ldots)$ & - \\
\hline
\end{tabular}


Distribución: Alemania: Aptiense inferior, biozona Deshayesites weissi (ver Koenen, 1902).

Francia: Cuenca Vocontiana, Aptiense inferior (Fallot, 1920). Barremiense superior-Aptiense inferior (Delanoy, 1992). Aptiense inferior, biozona Deshayesites weissi (ver Delanoy, 1997). Aptiense inferior, biozonas Deshayesites deshayesi, Dufrenoyia furcata (ver Dutour, 2005).

Eslovaquia: Barremiense superior y Barremiense-Aptiense inferior (Vašíček, 1972).

España: Cuenca d'Organyà: Aptiense inferior, biozona Deshayesites deshayesi (ver Martínez, 1982). Cuenca del Maestrazgo: Aptiense inferior, biozona Deshayesites forbesi (=Deshayesites weissi) (ver Murat, 1983). Aptiense inferior del Prebético de Alicante (Lillo, 1975). Aptiense inferior, Subbético (Braga et al., 1982).

Georgia: Barremiense superior-Aptiense inferior (Rouchadzé, 1933).

Túnez: Aptiense inferior (Lehmann et al., 2009).

? Polonia: Barremiense superior-Aptiense inferior (Ksiąźkiewicz, 1938).

? México: Aptiense inferior (González-Arreola et al., 1996).

Yacimientos: La Tejería de Josa, Km. 8,6 de la carretera Oliete-Muniesa.

\section{BIOESTRATIGRAFÍA}

Actualmente las biozonas de ammonites del Aptiense inferior se basan en distintas especies de deshayesítidos (Reboulet et al., 2009) por su rápida evolución, mientras que las distintas especies de desmocerátidos, de más amplia distribución estratigráfica son consideradas de poco valor bioestratigráfico. En los últimos años, se han realizado numerosos trabajos bioestratigráficos sobre ammonites del Aptiense inferior en distintas cuencas europeas, lo que ha permitido conocer con más detalle la distribución de las especies índices y de las especies acompañantes.
En la subcuenca de Oliete, los deshayesítidos tienen una presencia muy escasa y por tanto es de especial interés conocer con precisión la distribución de estas especies acompañantes, como los desmocerátidos.

Hasta Casey (1961) no hay referencias biostratigráficas precisas sobre desmocerátidos como Pseudosaynella cf. fimbriata Imlay, 1945 y Pseudosaynella aff. undulada (Sarasin, 1893) que atribuye a la biozona de Deshayesites forbesi, subzona de Deshayesites callidiscus (provincia faunística boreal), equivalente a la parte media/alta de la biozona Deshayesites weissi (Reboulet et al., 2009) en la provincia mediterránea. Murat (1983) reconoce Pseudosaynella josaensis Sornay \& Marin, $1972(=P$. raresulcata) en la biozona Deshayesites forbesi. Guérin-Franiatte (in Fischer \& Gauthier, 2006) y Busnardo \& Gauthier (in Fischer \& Gauthier, 2006) en la revisión sistemática de las especies de la colección d'Orbigny, incluyen Pseudosaynella bicurvata y $P$. raresulcata en las biozonas mediterráneas, en la de Deshayesites weissi y en la de Deshayesites deshayesi. Moreno et al. (2007) citan Pseudosaynella bicurvata, $P$. raresulcata y $P$. undulata en la parte alta de la biozona Deshayesites weissi (véase Fig. 5). Martínez (1982) y Martínez et al. (1994) citan Pseudosaynella bicurvata, P. raresulcata y $P$. undulata en la biozona Deshayesites deshayesi. Moreno-Bedmar et al. (2009, en prensa) que calibran la edad del Oceanic Anoxic Event la (OAE 1a) mediante los ammonoideos del Aptiense inferior de la Cuenca del Maestrazgo (E de España), atribuyen las especies Pseudosaynella bicurvata, $P$. raresulcata y $P$. undulata a la parte media/alta de la biozona Deshayesites weissi y a la biozona Deshayesites deshayesi. Recientemente, algunos de los autores del presente trabajo, han identificado la presencia de Pseudosaynella sp. en la parte baja de la biozona Dufrenoyia furcata en la subcuenca del Perelló (NE de España).

En consecuencia, la primera aparición de las especies del género Pseudosaynella se encuentra en la parte media/alta de la biozona Deshayesites weissi $(=$ Deshayesites forbesi, subzona Deshayesites callidiscus) y estas formas continúan estando presentes en la biozona suprayacente

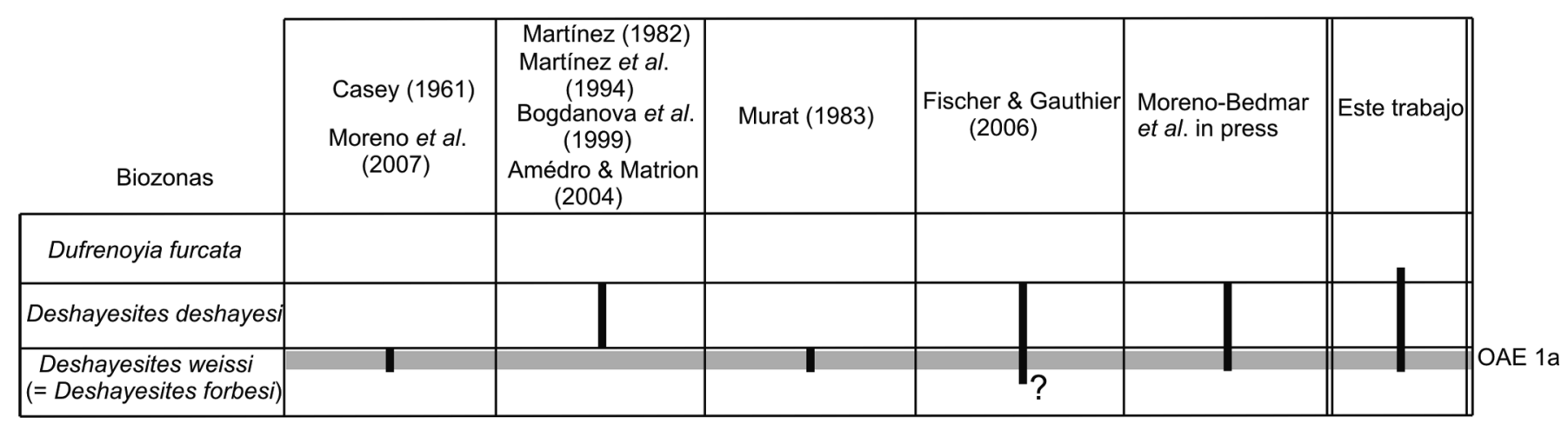

Figura 5. Rango de distribución bioestratigráfica de las especies Pseudosaynella. En gris el Evento Oceánico Anóxico 1a (OAE 1a). Range of the species Pseudosaynella. In grey the Oceanic Anoxic Event 1a (OAE 1a). 
Deshayesites deshayesi y en la parte baja de la biozona Dufrenoyia furcata (Fig. 5). Asimismo la primera aparición de las especies del género Pseudosaynella se halla justo antes del OAE 1a. La primera aparición posee pues un marcado valor bioestratigráfico.

Los datos bioestratigráficos existentes sobre las especies de Pseudohaploceras muestran distribuciones muy amplias, con lo que su aplicación bioestratigráfica no parece posible.

\section{AGRADECIMIENTOS}

Este trabajo ha sido financiado por los proyectos CGL200804916 y CGL2007-60054 ambos del Ministerio de Educación y Ciencia y de los grupos consolidados de investigación "Geologia Sedimentària" (2009SGR 1451) de la Generalitat de Catalunya y "Grup del Cretàci y Terciari inferior" (2005SGR 00320) de la Generalitat de Catalunya. Agradecer a José María Quer y Albert Casasús que nos permitieran estudiar los sus coleciones particulares.

\section{REFERENCIAS}

Abu-Zied, R.H. 2008. Lithostratigraphy and biostratigraphy of some Lower Cretaceous outcrops from Northern Sinai, Egypt. Cretaceous Research, 29, 603-624.

Amédro, F. \& Matrion, B. 2004. Les ammonites aptiennes des argiles à Plicatules de la Bordure orientale du bassin de Paris: un aperçu. Bulletin Association Géologique Auboise, 24-25, 75-80.

Bogdanova, T.N. \& Hoedemaeker, Ph.J. 2004. Barremian-Early Albian Deshayesitidae, Oppeliidae, Desmoceratidae and Silesitidae of Colombia. Scripta Geologica, 128, 183-312.

Bogdanova, T.N. \& Prozorosvky, V.A. 1999. Substantiation of the Barremian/Aptian boundary. Scripta Geologica, Special Issue 3, 45-81.

Braga, J.C., Company, M., Linares, A., Rivas, P. \& Sandoval, J. 1982. Contribución a la bioestratigrafía del Aptense de las Cordilleras Béticas. Cuadernos de Geología ibérica, 8, 691-701.

Canérot, J., Cuny, P., Pardo, G., Salas, R. \& Villena., J. 1982. Ibérica Central-Maestrazgo. In: El Cretácico de España. Universidad Complutense de Madrid, 273-344.

Casey, R. 1961. A monograph of the Ammonoidea of the Lower Greensand. Part 3. Monograph of the Paleontographical Society, 115, 119-216.

Chenu, J.C. 1842. Illustrations conchyliologiques ou description et figures de toutes les coquilles connues vivantes et fossiles, classées suivant le système de Lamarck modifié d'après les progrès de la science et comprenant les genres nouveaux et les espèces récemment découvertes. A. Frank Éditeur, Paris, 212 pls.

Delanoy, G. 1992. Les ammonites du Barremien Supérieur de Saint-Laurent de l'Escarène (Alpes-Maritimes, Sud-Est de la France). Annales du Muséum d'Historie Naturelle de Nice, 9, 1-148.

Delanoy, G. 1997. Bioestratigraphie des faunes d'Ammonites à la limite Barrémien-Aptien dans la région d'Angles-Bar-
reme-Castellane. Annales du Museum d'Historie Naturelle de Nice, 12, 1-270.

Dimitrova, N. 1967. Les fossiles de Bulgarie IV. Le Crétacé inférieur. Céphalopodes (Nautiloidea et Ammonoidea). Académie Bulgare des Sciences, Sofia, 236 pp. (En búlgaro).

Douvillé, R. 1906. Esquisse géologique des Préalpes Subbétiques (partie centrale). Tesis Doctoral, Université de Paris, $222 \mathrm{pp}$.

D’Orbigny, A. 1840-1842. Paléontologie française. Description zoologique et géologique de tous les animaux mollusques et rayonnés fossiles de France. Terrains Crétacés. Vol. 1. Céphalopodes. Arthus-Bertrand; Orbigny, Paris, $662 \mathrm{pp}$. [1-120 (1840); 121-430 (1841); 431-662 (1842)], $148 \mathrm{pl}$.

Drushchits, V. 1960. Ammonitii. In: Atlas Nizhnemelovoi fauny severnogo Kavkaza i Kryma (eds. V. Drushchits \& M. P. Kudriavtsev). Vsesoiuznyi Nauchno-Issledovatel'skii Institut Prirodnykh Gasov, Moskva, 249-355 (en ruso).

Dutour, I. 2005. Biostratigraphie, évolution et renouvellements des ammonites de l'Aptien supérieur (Gargasien) du bassin vocontien (Sud Est de la France). Tesis doctoral, Université Claude Bernard-Lyon, 278 pp. (inédita)

Fallot, P. 1920. Observations sur diverses espèces du Gargasien bathyal alpin et en particulier sur la faune de Blieux. In: Contributions à l'étude des céphalopodes paléocrétacés du Sud-Est de la France (Dir. W. Kilian). Mémoires pour servir à l'explication de la Carte géologique detaillée de la France, Paris, 229-266.

Fischer, J.C. \& Gauthier, H. 2006. Révisión crítique de la Paléontologie Françáise d'Alcide d'Orbigny, Volume IV, Céphalopodes Crétacés. Backhuys, Leiden, 292 pp.

González-Arreola, C., Pantoja-Alor, J., Olóriz, F., Villaseñor, A.B. \& García-Barrera, P. 1996. Lower Aptian Ammonitina Pseudohaploceras liptoviense (Zeuschner) in the Cumburindio Formation (Southwestern Mexico). Geobios, 29, 35-43.

Hill, R.T. 1888. The Neozoic geology of southwestern Arkansas. Arkansas Geological Survey, Annual Report, 2, 1-188.

Imlay, R.W. 1945. Ammonites from the Dierks limestone of southern Arkansas. Journal of Paleontology, 19, 277-281.

Kilian, W., 1913. Das bathyale Palaeocretacicum im südöstlichen Frankreich. Valendis-Stufe; Hauterive-Stufe; Barrême-Stufe, Apt-Stufe. Lethaea geognostica. Das Mesozoicum, 3. Kreide. III, Stuttgart, 289-398.

Koenen, A. von. 1902. Die Ammonitiden des Norddeutschen Neocom (Valanginien, Hauterivien, Barremien und Aptien). Abhandlungen der Königlich Preussischen Geologischen Landesanstalt und Bergakademie (Neue Folge), 24, 451 pp.

Ksiąźkiewicz, M. 1938. Fauna górno-neokomska z Lanckorony. Sprawozdania Komisji Fizyograficnnej, 72, 223-261.

Lehmann, J., Heltdt, M., Bachmann, M. \& Hedi Negra, M.E. 2009. Aptian (Lower Cretaceous) biostratigraphy and cephalopods from nordh central Tunisia. Cretaceous Research, 30, 895-910.

Leymerie, M.A. 1842. Suite du mémoire sur le terrain Crétacé du Département de l'Aube. Mémoires de la Société Géologique de France, 5, 1-34.

Lillo, J. 1975. Sobre algunos Desmocerataceae (Ammonitina) del Cretácico Inferior del sur de Alicante. Estudios Geológicos, 31, 681-704. 
Luppov, N.P., Bodylevsky, V.I. \& Glazunova, A.E. 1949. Class Cephalopoda. In: Atlas of index forms of the fossil faunas of the USSR, vol. 10. Lower Cretaceous (Ed. N.P. Luppov). Gosgeolizdat, Moskva \& Leningrad, 328 pp. (en ruso).

Marin, P. \& Sornay, J., 1971. Précisions sur l'âge des formations aptiennes aux confins de l'Aragón et du Maestrazgo (Provinces de Teruel et Castellón de la Plana, Espagne). Comptes Rendus sommaires de la Société géologique de France, 1971, 165-167.

Martínez, R. 1982. Ammonoideos cretácicos del Prepirineo de la provincia de Lleida. Universidad Autónoma de Barcelona. Publicaciones de Geología, 17, 197 pp.

Martínez, R. \& Grauges, A. 2006. Nautílidos del Aptiense Inferior (Cretácico Inferior) de la Subcuenca de Oliete, Cordillera Ibérica oriental (Teruel, España). Revista Española de Paleontología, 21, 15-27.

Martínez, R., Grauges, A. \& Salas, R. 1994. Distribución de los ammonites del Cretácico inferior de la Cordillera Costera Catalana e Ibérica Oriental. Cuadernos de Geología Ibérica, 18, 337-354.

Michelin, H. 1838. Note sur une argile dépendant du Gault. Mémoires de la Société Géologique de France, 3, 97-103.

Moreno, J.A., Company, M., Delanoy, G., Grauges, A., Martínez, R. \& Salas, R. 2007. Precisiones sobre la edad, mediante ammonoideos y nautiloideos, de la Fm. Margas del Forcall en la subcuenca de Oliete (Cadena Ibérica, España). Geogaceta, 42, 75-78.

Moreno-Bedmar, J.A., Company, M., Bover-Arnal, T., Salas, R., Delanoy, G., Martínez, R. \& Grauges, A. 2009. Biostratigraphic characterization by means ammonoids of the Lower Aptian Oceanic Anoxic Event (OAE 1a) in the Eastern Iberian Chain (Maestrazgo Basin, eastern Spain). Cretaceous Research, 30, 864-872.

Moreno-Bedmar, J.A., Company, M., Delanoy, G., Maurrasse, F., Bover-Arnal, T., Salas, R., Grauges, A. \& Martínez, R. en prensa. Lower Aptian ammonite biostratigraphy in the Maestrazgo basin (Eastern Iberian chain, Spain). Geologica Acta.

Murat, B. 1983. Contribution à l'étude stratigraphique, sédimentologique et tectonique du bassin éocrétace d'Oliete (Prov. de Teruel, Espagne). Doctoral thesis. Travaux du Laboratoire Géologie Sédimentologie et Paléontologie, Université Paul Sabatier, Tolouse, 247 pp.

Orlov, Y.A. (ed.) 1958. Principles of paleontology. 6, Mollusca: Cephalopoda, II, Ammonoidea (ceratites and ammonites), Dibranchiata. Ed. Gosudarstvennoe Nauchno-Tekhnicheskoe Izdatet'stvo Literatury po Geologii i Okhrane Nedr., Movska, 359 pp. (En ruso)

Orlov, Y.A. (ed.) 1976. Fundamentals of paleontology. 6, Mollusca: Cephalopoda, II, Ammonoidea (Ceratitida and Ammonitida), Endocochlia, Coniconchia. Program for Scientific Translations, Jerusalem, 474 pp. (traducción del original ruso: Orlov, 1958)

Reboulet, S., Klein, J., Barragán, R., Company, M., GonzálezArreola, C., Lukeneder, A., Raisossadat, S.N., Sandoval, J., Szives, O., Tavera, J.M., Vašíček, Z. \& Vermeulen, J. 2009. Report on the 3rd International Meeting of the IUGS Lower Cretaceous Ammonite Working Group, the "Kilian
Group" (Vienna, Austria, 15th April, 2008). Cretaceous Research, 30, 496-502.

Rouchadzé, J. 1933. Les ammonites aptiennes de la Géorgie occidentale. Bulletin de l'Institute Geologique de Georgie, 1, 165-273.

Salas, R. \& Guimerà, J. 1996, Rasgos estructurales principales de la cuenca cretácica inferior del Maestrazgo (Cordillera Ibérica oriental). Geogaceta, 20, 1704-1706.

Salas, R., Guimerà, J., Martín-Closas, C., Meléndez, A., \& Alonsos, A. 2001. Evolution of the Mesozoic Central Iberian Rifting System and its Cainozoic inversion (Iberian Chain). In: Peri-Tethys Memoir 6: Peri-Tethyan Rifting/ Wrench Basins and Passive Margins (eds. P. A. Ziegler, W. Cavazza, A.H.F. Roberston \& S. Crasquin-Soleau). Mémoires du Muséum National d'Histoire Naturelle, Paris, 186, 145-186.

Sarasin, Ch. 1893. Étude sur les Oppelia du groupe du Nisus et les Sonneratia du groupe du Bicurvatus et du Raresulcatus. Bulletin de la Société Géologique de France, sèrie $3^{\text {eme }} \mathbf{2 1}, 149-164$.

Schloenbach, U. 1868. Kleine paläontologische Mittheillungen. Jahrbuch der Geologischen Bundesandstalt, 18, 465-468.

Sornay, J. \& Marin, P. 1972. Sur la faune d'ammonites aptiennes de la Tejería de Josa (Teruel, Espagne). Annales de Paléontologie, 58, 101-123.

Tietze, E. 1872. III Geologische und paläontologische Mittheilungen aus dem südlichen Teil des Banater Gebirsstockes. Jahrbuch der Kaiserlich-Königlichen Geologischen Reichsanstalt, 22, 35-142.

Trautschold, H. 1865. Die Innoceramen-Thon von Simbirsk. Bulletin de la Société Imperiale des Naturalistes de Moscou, 38, 1-24.

Uhlig, V. 1883. Die Cephalopodenfauna der Wernsdorfen Schichten. Denkschriften der Kaiserlichen Akademie der Wissenschaften, Wien, Mathematisch-Naturwissenschaftliche Klasse, 46, 127-290

Vašíček, Z. 1972. Ammonoidea of the Těšín-Hradiště Formation (Lower Cretaceous) in the Moravskoslezské Beskydy Mts. Ústředního ústavu geologického, 38, 107 pp.

Vilanova, J. 1870. Ensayo de descripción geognóstica de la Provincia de Teruel en sus relaciones con la agricultura de la misma. Junta de Estadística, Madrid, años 1863, 1868, 312 pp.

Waitzman, S. 1960. Contribution à l'étude des Ammonites du Crétacé inférieur d'Espagne. Diplome d'Etudes superieures. Universidad de Paris, 109 pp. (Inédito)

Wright, C.W., Callomon, J.H. \& Howarth, M.K. 1996. Mollusca 4 (Revised) Cretaceous ammonoidea, Part L. In: Tretaise on Invertebrate Paleontology (Ed. R. Kaesler). Geological Society of America/ University of Kansas Press, Boulder/Lawrance, $362 \mathrm{pp}$.

Zeuschner, L. 1856. Geognostische Beschreibung des Liaskalkes in der Tatra und in den angrenzenden Gebirgen. Sitzungsberichte der Kaiserlichen Akademie der Wissenschaften in Wien, 19, 135-182.

Manuscrito recibido: 24 de Julio, 2009 Manuscrito aceptado: 22 de Marzo, 2010 